\title{
Downregulation Of microRNA-133b And Its Clinical Value In Non-Small Cell Lung Cancer
}

This article was published in the following Dove Press journal: OncoTargets and Therapy

\section{Guan-yu Chen \\ Lin Ruan}

Departments of Anesthesiology, Affiliated Tumor Hospital of Guangxi Medical University, Nanning, Guangxi, People's Republic of China
Correspondence: Lin Ruan

Departments of Anesthesiology, Affiliated Tumor Hospital of Guangxi Medical

University, Nanning, Guangxi, People's

Republic of China

Email Ruanlin187@I63.com
Background: Previous studies have investigated the expression of miR-133b in non-small cell lung cancer (NSCLC); however, its underlying mechanism in relation to the pathogenesis of NSCLC remains unclear.

Methods: The aim of this study was to investigate the correlation between miR-133b expression and clinical parameters based on the Cancer Genome Atlas (TCGA) and realtime quantitative real-time PCR (RT-qPCR) data. Gene Ontology (GO) and Kyoto Encyclopedia of Genes and Genomes (KEGG) analyses were performed to identify the biological function of miR-133b. A protein-protein interaction (PPI) network was constructed to screen for hub genes. The Gene Expression Profiling Interaction Analysis (GEPIA) and the Human Protein Atlas databases (HPAD) were employed to validate the hub genes. The cBioPortal database was used to identify neighboring genes with alteration frequencies greater than $20 \%$ gene alterations.

Results: miR-133b was downregulated in NSCLC tissues, and expression was correlated with lymph node metastasis $(\mathrm{P}<0.05)$. A total of 362 genes were considered as the potential targets of miR-133b in NSCLC. These candidate target genes highly enriched in various key pathways such as the PI3K-Akt pathways, P53 signal pathways, and ECM-receptor interaction. PPI revealed 10 genes as hub genes with node degrees $\geq 10$.

Conclusion: The study validated that miR-133b is downregulated in NSCLC. In addition, miR-133b might function as a biomarker for the diagnosis and prognosis of NSCLC. Bioinformatics analysis revealed that miR-133b could be involved in NSCLC metastasis.

Keywords: miR-133b, non-small cell lung cancer, microarray, miRNA-sequencing, quantitative real-time PCR

\section{Background}

Lung cancer (LC) is considered a life-threatening disease, and its morbidity and mortality rank second among all tumors. ${ }^{1}$ Non-small cell lung cancer (NSCLC) accounts for $85 \%$ of all diagnosed LC cases. ${ }^{1}$ Currently, the main treatment of NSCLC is a combination of surgery and chemotherapy. ${ }^{2}$ Although early detection, diagnosis, and targeted therapy of NSCLC have made significant progress, the fiveyear survival rate is still very low. ${ }^{3}$ Regional or distant metastasis is a risk factor for relapse and poor prognosis of NSCLC. ${ }^{3}$ Therefore, there is an urgent need to identify novel markers to study the mechanism underlying the NSCLC recurrence and metastasis to improve patient survival rate.

MicroRNAs (miRNAs) are short endogenous non-coding RNAs that play an important role in the regulation of gene expression by binding to the mRNA of target genes after transcription. ${ }^{4}$ Recently, miRNAs have been considered as potential biomarkers for 
the diagnosis, treatment, and prognosis of cancer. There is increasing evidence that some miRNAs are abnormally expressed and play a key role in regulating the growth and metastasis of NSCLC. ${ }^{5,6}$ miR-133b has been shown to be a tumor suppressor that inhibits the progression of various cancers, including gastric, esophageal, lung cancer, and ovarian cancers. ${ }^{7-10}$ Therefore, miR-133b may be a promising biomarker of NSCLC. However, the mechanism of miR-133b in NSCLC metastasis and the relationship between miR-133b and clinical characteristics have not been fully elucidated.

We investigated the correlation between miR-133b expression and clinical parameters based on gene. The Cancer Genome Atlas (TCGA) and real-time quantitative real-time PCR (RT-qPCR) data. We then explored the potential mechanism of action of miR-133b in NSCLC by predicting genes targeted by miR-133b and performing enrichment analysis. In addition, we also screened for hub genes by constructing a protein-protein interaction (PPI) network and validated their expression in tissues in TCGA and immunohistochemistry. Finally, we selected the target genes and pathways of interest to discuss the possible mechanisms of miR-133b in NSCLC.

\section{Materials And Methods}

\section{MiRNA-133b Expression Data From TCGA}

TCGA (https://cancergenome.nih.gov/) was used to obtain expression values of miR-133b in NSCLC and normal tissues. Differences in miR-133b expression between NSCLC and normal tissues were calculated using $\mathrm{R}$ software (version 3.6.0).

\section{Quantitative Real-Time PCR}

A total of 44 paired non-small cell lung cancer specimens were obtained from the Affiliated Tumor Hospital of Guangxi Medical University, Nanning, China from January 2017 to December 2018 were collected for this study. The study methodologies conformed to the standards set by the Declaration of Helsinki, and the study was approved by the hospital's ethics committee. Written informed consent was obtained from the patients to utilize their tissue samples. Formalin fixation and paraffin embedding (FFPE) were performed to preserve the specimens. Next, total RNA of tissue samples was extracted using TRIzol ${ }^{\circledR}$ reagent (Invitrogen). After pipetting $1 \mu \mathrm{g}$ of RNA, a variety of reagents were added according to the instructions of M-MLV reverse transcriptase kit (TAKARA, Dalian, China). Quantitative real-time PCR was performed using Applied Biosystems 7900HT Fast Real-Time PCR
System software. PCR amplification was performed using primers designed with Primer Premier 5.0 software. The primers were as follows: has-miR-133b, forward, 5'-GAACC AAGCCGCCCGAGA-3' and reverse, 5'-CCGCCCTGCTGT GCTGGT-3'; $\beta$-actin, forward 5-GGGAAATCGTGCGTG ACATTAAG-3' and reverse 5'-TGTGTTGGCGTACAGG TCTTTG-3'. The expression levels of miR-133b was normalized to the expression of $\beta$-actin using the $2^{-\Delta \Delta C T}$ method. The expression of miR-133b in the NSCLC tissues of each patient was compared to that of the average of the adjacent normal tissues, and the patients were accordingly grouped into highand low-expression groups.

\section{Predictive Value Of miR-I33b In NSCLC Prognosis}

The prognostic roles of miR-133b in NSCLC were analyzed using TCGA data. Differences with a Cox $P<0.05$ were considered statistically significant.

\section{Diagnostic Value Of miR-I33b In The Diagnosis Of NSCLC}

To evaluate the diagnostic value of miR-133b in the diagnosis of NSCLC, the ROC curve of miR-133b in NSCLC was established using IBM SPSS Statistics V22.0 software. The area under the ROC curve $>0.5$ was considered to have a certain diagnostic value. At the same time, the closer the area under the ROC curve to 1 , the higher the diagnostic value of miR-133b for the diagnosis of NSCLC.

\section{Candidate Targets Of miR-I33b In NSCLC}

The target gene of miR-133b was predicted by miRWalk 3.0, which identifies miRNA binding sites within the complete sequence of a gene, but also combines this information with a comparison of binding sites resulting from 12 existing miRNA-target prediction programs. It is widely known that miRNAs exert their biological effect primarily by directly targeting the $3^{\prime}$ untranslated region of mRNA. To improve the reliability of our subsequent analysis of the screened miR-133b target genes, we next verified the expression of these target genes in the Gene Expression Profiling Interaction Analysis (GEPIA) database. The intersection of the upregulated genes in LUAD and LUSC with the predicted target genes was considered a target gene of miR-133b in NSCLC. 


\section{GO Annotation And KEGG Pathway Enrichment Analysis}

To identify the biological function of miR-133b, the Enrichr database (http://amp.pharm.mssm.edu/Enrichr/) was used to perform GO functional annotation and KEGG pathway enrichment analysis of the target genes and the most frequently altered neighboring genes. GO functional annotation analysis included three categories: biological process (BP), cellular component (CC), and molecular function (MF).

\section{PPI Network Analysis}

To better understand the relationship among the screened genes, a PPI network was established using STRING (https://string-db.org/). The hub genes were identified according to degree using Cytoscape (version 3.6.1).

\section{Validation Of Hub Genes}

The expression levels of the top 10 hub genes were further validated using the GEPIA and the Human Protein Atlas (https://www.proteinatlas.org/) databases. Hub genes with $|\log 2 \mathrm{FC}|>2$ and $\mathrm{P}<0.05$ were considered statistically significant.

\section{GEPIA Database Analysis}

The prognostic roles of screened hub genes in NSCLC were analyzed using the GEPIA database, which is a newly developed interactive web server for analyzing the RNA sequencing expression data of 9736 tumors and 8587 normal samples from the TCGA and the GTEx projects. Differences with a Cox $P<0.05$ were considered statistically significant.

\section{c-BioPortal Analysis}

Alterations involving hub genes in the NSCLC samples were analyzed using the c-BioPortal database. The tab OncoPrint displays an overview of genetic alterations in hub genes. The tab co-expression displays an overview of co-expressed of hub genes. The tab Network visualizes the biological interaction of hub genes, and includes neighboring genes with alteration frequencies $>20 \%$. We then performed GO and KEGG pathway enrichment analyses of the most frequently altered neighboring genes using the Enrichr database.

\section{Statistical Analysis}

The number of cancer and non-cancer groups, mean (M), and standard deviation (SD) of miR-133b in the TCGA databases were calculated using $\mathrm{R}$ software. Most of the statistical analysis were performed using bioinformatics tools as earlier mentioned. Differences with Cox $\mathrm{P}<0.05$ were considered as statistically significant for survival analysis.

\section{Results}

\section{Relationship Between miRNA-I33b Expression And Clinical Characteristic Based On TCGA Data}

TCGA included 444 samples for LUSC patients and 343 samples for LUAD patients. For LUSC, miR-133b expression was downregulated compared with normal tissues $(0.9882589$ \pm 0.6567950 vs. $2.5674577 \pm 0.7299547, \mathrm{p}<0.001$ [Figure 1B and Supplement Table 1]). For LUAD, the expression level of miR-133b was significantly lower than in normal tissues $(1.095345 \pm 0.7195799$ vs. $2.238352 \pm 0.7299547, \mathrm{p}<0.001$

[Figure 1A and Supplement Table 1]). The expression level of miR-133b was downregulated in NSCLC tissues compared with normal tissues $(1.049451 \pm 0.6949068$ vs. $2.5476656 \pm$ $0.7288576, \mathrm{p}<0.0001$ (Figure 1C and Supplement Table 1). A Kaplan-Meier curve was used to identify the effects of miR$133 \mathrm{~b}$ expression on survival rate. Figure 2 shows that the $p$ values of the Kaplan-Meier curves were $<0.05$ in LUAD, thus indicating significant differences in survival between the downregulated and upregulated miR-133b groups. The ROC curve of miR-133b in NSCLC is listed in Figure 3. The areas under the ROC curve $>0.5$ were $0.873,0.905$, and 0.937 for LUAD, LUSC, and NSCLC, respectively, indicating that miR$133 \mathrm{~b}$ could be considered as a diagnostic marker for NSCLC. Supplement Table 1 shows the clinical characteristics of 332 LUSC patients and 445 LUAD patients were downloaded from TCGA. Regarding LUAD and NSCLC, a significant difference in miR-133b was observed at the $\mathrm{N}$ stage $(\mathrm{p}=$ 0.01195 and $\mathrm{p}=0.01192$, respectively).

\section{Relationships Between miRNA-I33b Expression And Clinical Characteristics Based On qRT-PCT Analysis}

Figure 4 and Supplement Table 2 show that the NSCLC group has significantly lower miR-133b expression levels than the normal group $(0.3782883 \pm$ 0.4082107 vs. $3.0388487 \pm 0.6888178, \mathrm{p}<0.001)$. miR- 
A

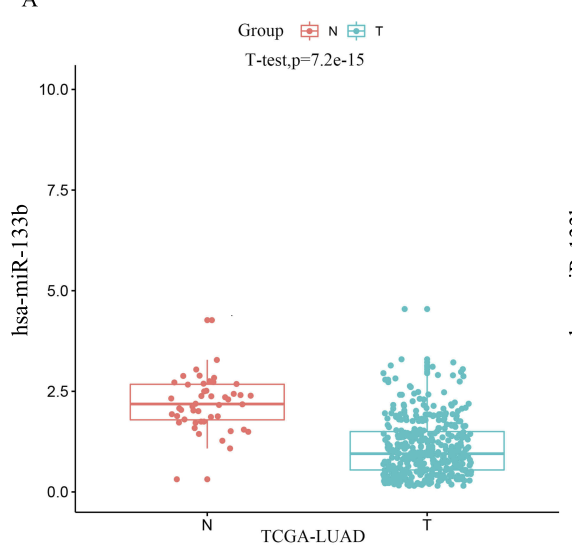

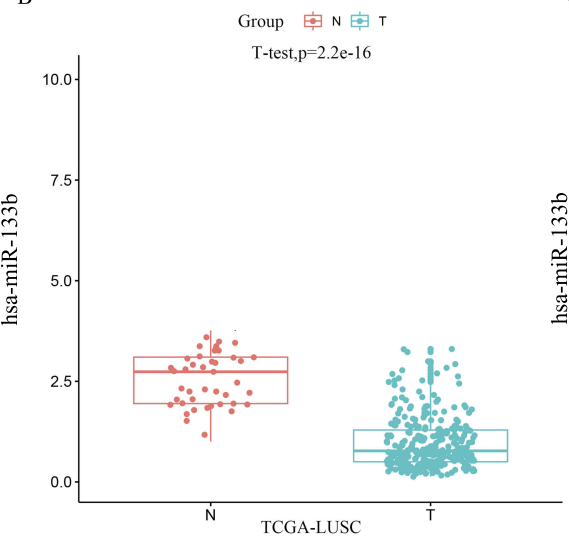

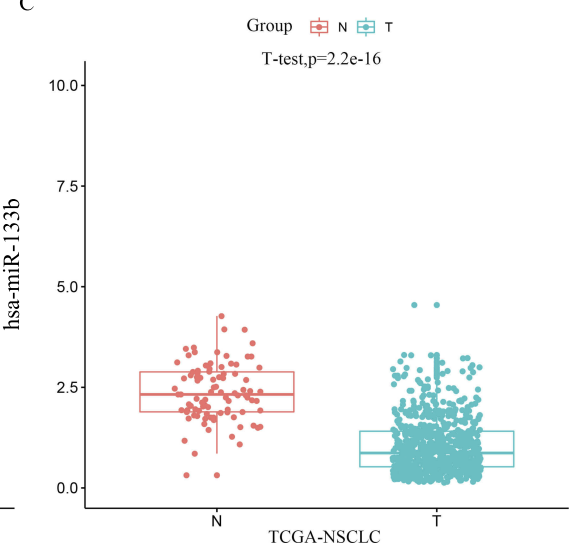

Figure I The expression of miRNA-I33b in NSCLC and control groups, based on The Cancer Genome Atlas data. (A) The expression of miRNA-I33b in LUAD; (B) The expression of miRNA-I33b in LUSC; (C) The expression of miRNA-I33b in NSCLC.
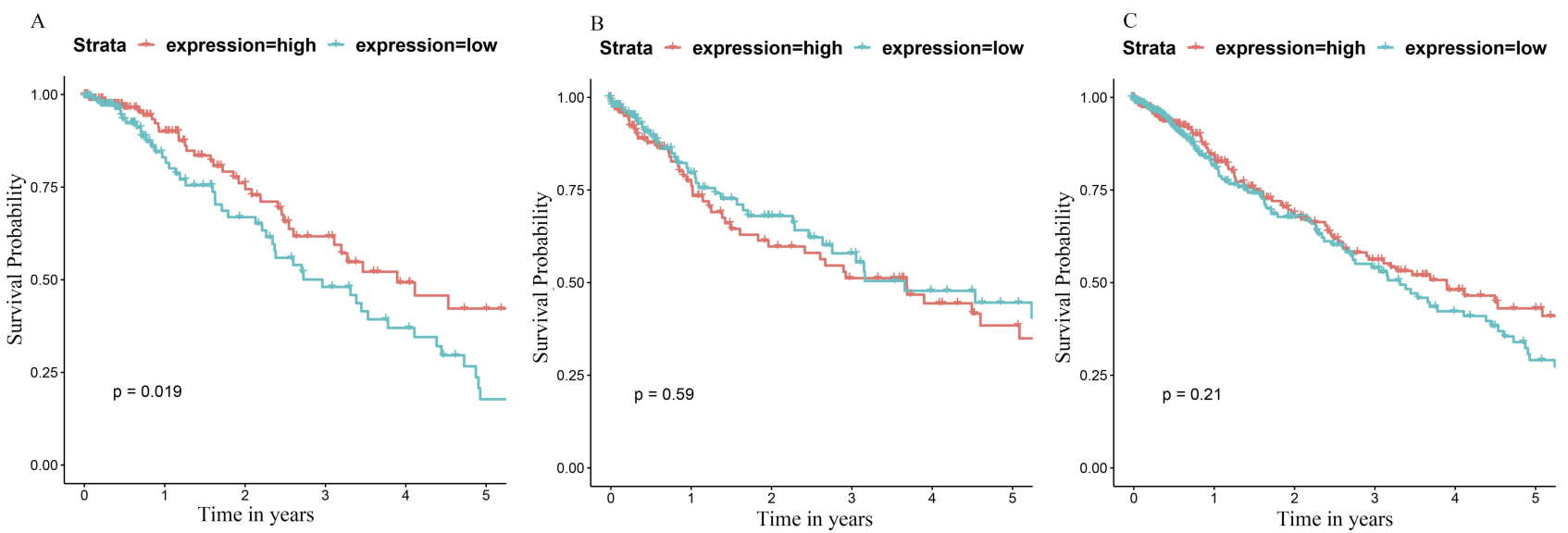

Figure 2 Kaplan-Meier curves for miRNA-I33b in NSCLC based on The Cancer Genome Atlas data. (A) Kaplan-Meier curves for miRNA-I33b in LUAD; (B) KaplanMeier curves for miRNA-I33b in LUSC; (C) Kaplan-Meier curves for miRNA-I33b in NSCLC. Cox $P<0.05$ was considered as significant.
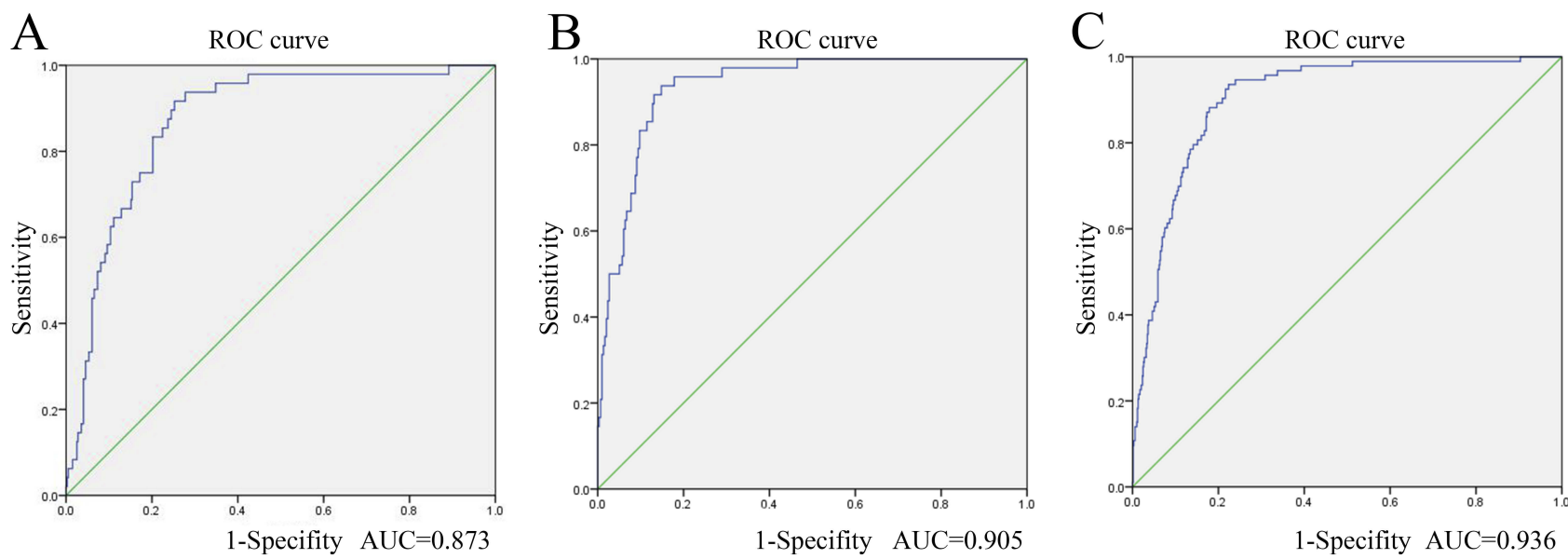

Figure 3 ROC curve for miR-133b in diagnosis of NSCLC. (A) ROC curve for miR- I33b in diagnosis of LUAD, AUC=0.873; (B) ROC curve for miR-I33b in diagnosis of LUSC, $A \cup C=0.905$; (C) ROC curve for miR-133b in diagnosis of NSCLC, AUC $=0.936$. The area under the ROC curve $>0.5$ was considered as to have a certain diagnostic value.

133b downregulation was observed in both LUSC and lymph node metastasis but not in those without $(\mathrm{p}=$ LUAD ( $\mathrm{p}<0.0001, \mathrm{p}<0.0001$, respectively). miR- 0.008; Supplement Table 2). miR-133b downregulation $133 \mathrm{~b}$ downregulation was observed in patients with was observed in males and LUSC relative to females 

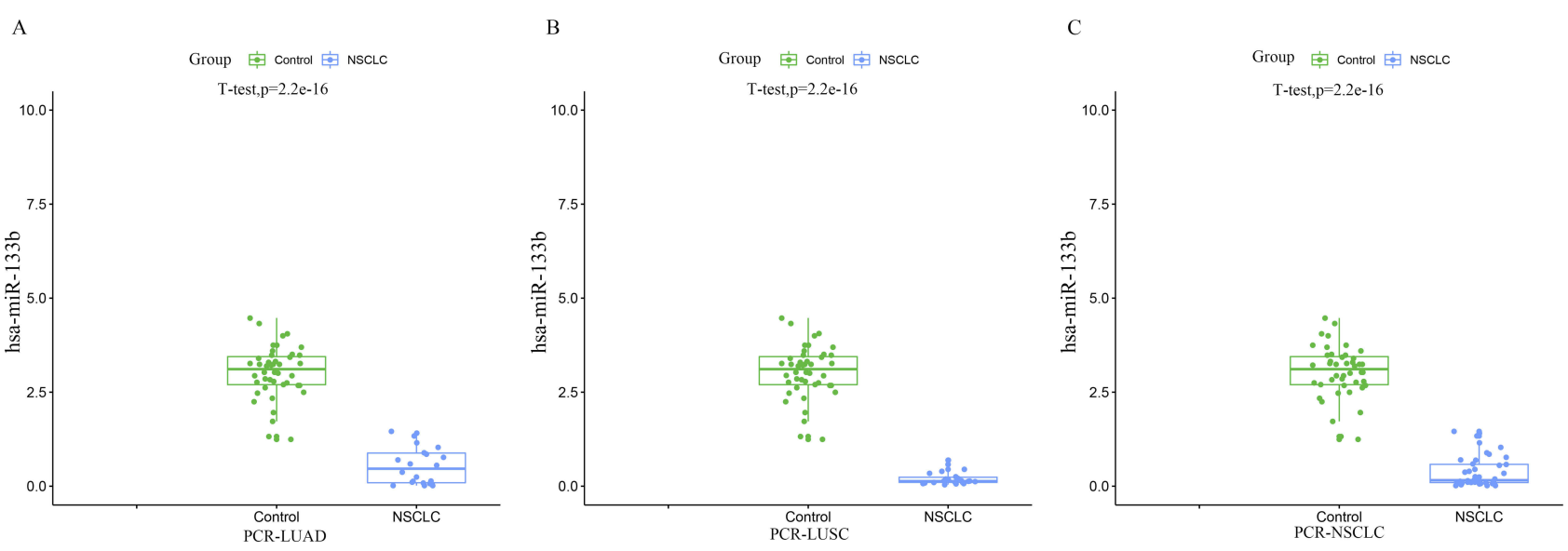

Figure 4 The expression of miRNA-I33b in NSCLC and control groups, based on quantitative real-time PCR data. (A) The expression of miRNA-I33b in LUAD and control groups; (B) The expression of miRNA-I33b in LUSC and control groups; (C) The expression of miRNA-I33b in NSCLC and control groups.

and LUSC ( $\mathrm{p}=0.009, \mathrm{p}=0.006$, respectively). No statistical differences in clinical stage, tumor size, smoking status, vascular invasion, or histology were observed (Supplement Table 2).

\section{Prediction Of Target Genes Of miR-I33b} miRWalk 3.0 predicted approximately 11,994 genes that are targeted by miR-133b in NSCLC. A total of 1111 and 1922 overexpressed genes in LUAD and LUSC were collected on the basis of GEPIA, respectively. After intersection of the upregulated genes in LUAD and LUSC with the predicted target genes, 362 predicted target genes were selected (Figure 5).

\section{GO Function And KEGG Pathway Enrichment Analyses}

The top 10 enriched GO items are listed in Figure 6. GO BP analysis revealed that the target genes of miR-133b significantly enriched the functional categories of DNA metabolic process and centromere complex assembly (Figure 6A). For CC analysis, these genes significantly enriched the functional categories of spindle and chromosome and centromeric region (Figure 6B). MF analysis of these genes revealed enrichment of the categories of protein binding involved in heterotypic cellcell adhesion and microtubule motor activity (Figure 6C).

KEGG pathway enrichment analysis of target genes was further performed. Figure $6 \mathrm{D}$ shows that the target genes of miR-133b significantly enriched the functional categories of cell cycle, oocyte meiosis, p53 signaling pathway, ECMreceptor interaction, progesterone-mediated oocyte maturation, mucin-type o-glycan biosynthesis, DNA replication, arrhythmogentic right ventricular cardiomyopathy, arginine and proline metabolism, and drug metabolism pathway.

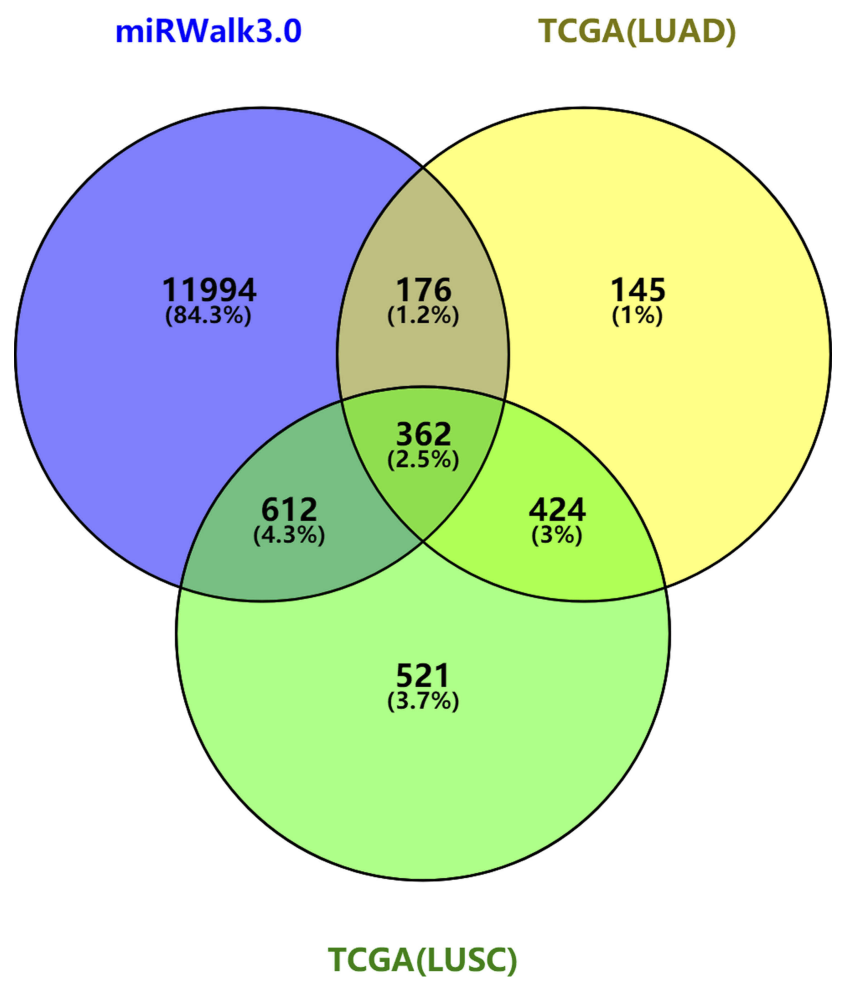

Figure 5 Venn diagram of target genes of miR-|33b.

\section{Screening Of Hub Genes}

We constructed a PPI network of these genes (Figure 7). To obtain the hub genes in the PPI network, these node pairs were entered into the Cytoscape software. The hub genes with a node degree value $\geq 10$ are listed in Supplement Table 3. For the target genes of miR-133b, the hub genes were STMN1, HIST1H2BD, POSTN, MMP1, H1F0, KIF26B, ITGB4, TMED3, POC1A, and CXCL10. 


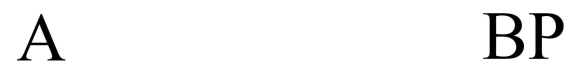

DNAmetabolic process (GO:0006259)

centromere complex assembly (GO:0034508)

mitotic sister chromatid segregation (GO:0000070)

chromatin remodeling at centromere (GO:0031055)

DNAreplication (GO:0006260)

kinetochore organization (GO:0051383)

CENP-Acontaining nucleos ome ass em bly (GO:0034080)

CENP-Acontaining chromatin organization (GO:0061641)

mitotic spindle organization (GO:0007052)

extracellular matrix organization (GO:0030198)

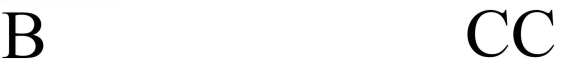

spindle (GO:0005819)
chrom os ome, centromeric region (GO:00
mitotic spindle (GO:0072686)
chrom os omal region (GO:0098687)
microtubule (GO:0005874)
spindle microtubule (GO:0005876)
nuclear chromos ome part (GO:0044454)
kinesin complex (GO:0005871)
microtubule cytoskeletor (GO:0015630)
spindle pole (GO:0000922)

$\mathrm{C} \quad \mathrm{MF}$

protein binding involved in heterotypic cell-cell adhesion (GO:0086080)

microtubule motor activity (GO:0003777)

motor activity (GO:0003774)

GPI anchor binding (GO:0034235)

collagen binding (GO:0005518)

tubulin binding (GO:0015631)

microtubule binding (GO:0p08017)

metalloendopeptidase activity (GO:0004222)

3'-5' DNAhe licase activity (GO:0043138)

cadherin binding (GO:0045296)

D $\quad$ KEGG

Cell cycle

Oocyte meiosis

p53 signaling pathway

ECM-receptor interaction

Progesterone-mediated oocyte maturation

Mucin type O-glycan biosynthesis

DNAreplication

Arrhythmogenic right ventricular cardiomyopathy (ARVC)

Arginine and proline metabolism

Drug metabolism

Figure 6 GO functional annotation and KEGG pathway analysis of candidate genes. (A) the top I0 enriched BP items of candidate genes; (B) the top I0 enriched CC items of candidate genes; (C) the top 10 enriched MF items of candidate genes; (D) The top 10 enriched KEGG pathways for the intersection of target genes of miR-I33b. 


\begin{tabular}{|c|c|c|c|c|c|c|c|c|c|c|c|c|c|c|c|c|}
\hline SPAG5 & GJB3 & CXCL14 & EXO1 & CENPN & MRPS35 & AK4 & IGSF9 & KIFC1 & SPTBN2 & ORC1 & GCNT3 & CLDN1 & HAMP & CDC6 & STRAG & $\mathrm{CDCP} 1$ \\
\hline DLGAP5 & PDIA6 & TPX2 & IQGAP3 & VTCN1 & FUT8 & $\mathrm{F} 12$ & NME2 & KPNA2 & STMN1 & GSTA1 & GREM1 & $\mathrm{CP}$ & ALDH3B2 & PPIL1 & PITX1 & SNRPE \\
\hline HMGA1 & LAMB3 & $\mathrm{S} 100 \mathrm{~A} 2$ & MMP12 & CCNF & MEX3A & MIS18A & PTRH2 & DDR1 & STEAP3 & STAT1 & HPRT1 & PAK1 & MARCKSL1 & PTPRF & ARHGEF 35 & DCTPP1 \\
\hline $\mathrm{SPC} 24$ & SULF1 & CENPF & SHCBP1 & HS6ST2 & MMP1 & MXRA5 & YWHAZ & MRPL24 & TXNDC17 & RAD51AP1 & TROAP & GPX2 & TNS4 & XDH & PRC1 & PDIA4 \\
\hline $\mathrm{CDC} 25 \mathrm{C}$ & FABP6 & GALNT14 & GRHL2 & C19orf48 & TMPRSS11E & THNSL1 & TACSTD2 & PITX2 & PTGES & NXT2 & S100A14 & ENO1 & MCM4 & $\mathrm{KIF} 20 \mathrm{~A}$ & CHMP4C & LMNB1 \\
\hline GINS2 & TMED10 & HMGB3 & UBD & MMP11 & CCNE1 & NCAPG & FNDC1 & THY1 & VPREB3 & PMAIP1 & FHL2 & KIF26B & PLAU & SDC1 & ORC6 & STIL \\
\hline HMMR & AMACR & AURKA & RHNO1 & CXCL10 & $\mathrm{CDH} 1$ & NQO1 & KIF15 & FAM83H & LAMC2 & ILDR1 & CHAF1B & CDK1 & АРОВЕСЗВ & $\mathrm{KIF} 4 \mathrm{~A}$ E & G0000025924 & CBLC \\
\hline POSTN & TGFA & SFN & METTL1 & HIST1H2BD & B3GNT3 & TK1 & ERGIC2 & ZWINT & KRT19 & CHEK1 & PTTG1 & PSAT1 & TMED3 & SPP1 & PABPC1 & сст3 \\
\hline SLC52A3 & TYMS & COL11A1 & NEK2 & TFAP2C & WDHD1 & ACN9 & FANCF & NCAPG2 & ARHGEF19 & IL411 & CENPL & EFNA3 & FGF11 & SUSD4 & NUF2 & SORD \\
\hline NGEF & TMEM132A & RASAL1 & CCNB2 & TRUB1 & FAM111B & TOP2A & CDCA3 & TMCO1 & CXCL9 & CD79A & CPXM1 & LDHA & MCM2 & PARPBP & DEPDC1 & CEACAM5 \\
\hline CENPW & MND1 & GPT2 & KDF1 & TONSL & RPP40 & $\mathrm{KIF} 2 \mathrm{C}$ & PFKP & NCAPH & TPBG & GEMIN6 & CCNB1 & ASPM & sox4 & EFNA4 & LYPD1 & FEN1 \\
\hline KRT8 & MRPL15 & UBE2T & OTX1 & TMPRSS4 & HOXB7 & SLC7A5 & H1FO & OIP5 & MMP9 & NUDT1 & PSMG3 & PLA2G2D & GPRIN1 & SLC12A8 & $\mathrm{CDC} 45$ & ADAM12 \\
\hline scG5 & KIF18B & PKMYT1 & CENPI & C3orf14 & SLC9A7 & STYK1 & SNRPD1 & DHFR & JUP & KRT16 & KIF11 & THOC3 & CKMT1A & $\mathrm{CDH} 3$ & SPAG4 & CKMT1B \\
\hline HELLS & DERL3 & UBE2S & SCAMP5 & CENPH & PBK & CKS1B & ITGA11 & SLC39A4 & BRIX1 & CDT1 & SKA1 & ANLN & DSP & MAD2L1 & CTHRC1 & ETV4 \\
\hline MYBL2 & ALDH18A1 & PC & ESRP1 & NUSAP1 & EPHB2 & EXOSC5 & PDZD11 & ADAMDEC 1 & CRABP2 & KRT80 & COL3A1 & LEPREL4 & SIX1 & RHOV & CENPA & CDCA5 \\
\hline ST14 & SMIM15 & SEMA4B & SPINT1 & CXCL13 & CA9 & PAFAH1B3 & $\mathrm{CDC} 20$ & KCNK1 & IGLL5 & POC1A & KRT15 & FOXM1 & ITGB4 & GINS1 & GGCT & sox2 \\
\hline PYCR1 & SLC39A11 & cCT5 & WNT7B & $\mathrm{DIO} 2$ & IRF6 & FANCI & UHRF1 & DCAF13 & PRPS2 & PPAT & NPM3 & COL1A1 & ECE2 & TMPRSS13 & RNASEH2A & $\mathrm{DSC2}$ \\
\hline RHGAP $11 \mathrm{~A}$ & FOXP3 & UGDH & E2F8 & SAPCD2 & GALNT6 & & & & & & & & & & & \\
\hline
\end{tabular}

Figure 7 The protein-protein interaction (PPI) networks of the promising target genes of microRNA-133b in non-small cell lung cancer.

\section{Confirmation Of Potential Hub Genes}

The GEPIA database was used to detect the expression levels of 10 hub genes. Figure 8 shows that the expression levels of the 10 hub genes were significantly lower in the NSCLC tissues than in normal tissues. In addition, Human Protein Atlas database (https://www.proteinatlas.org/) was used to validate the translational levels of the 10 hub genes (Figure 9). Immunohistochemical analysis of NSCLC sections revealed that most of the hub genes (MMP1 and CXCL10 were not available in the database) have moderate to high levels of expression. To further identify hub genes, the prognostic roles of these 10 hub genes in NSCLC were evaluated using the GEPIA database. Figure 10 shows that the higher expression of ITGB4 and POSTN is correlated to poor prognosis in NSCLC.

\section{Biological Interaction Network Of Hub Gene Alterations In NSCLC}

cBioPortal was used to show neighboring genes that were altered at frequencies $>20 \%$ (Figure $11 \mathrm{~A}$ and $\mathrm{B}$ ). The 10 hub genes were altered in NSCLC, and KIF26B, $S T M N 1$, and POSTN were altered most often in LUAD
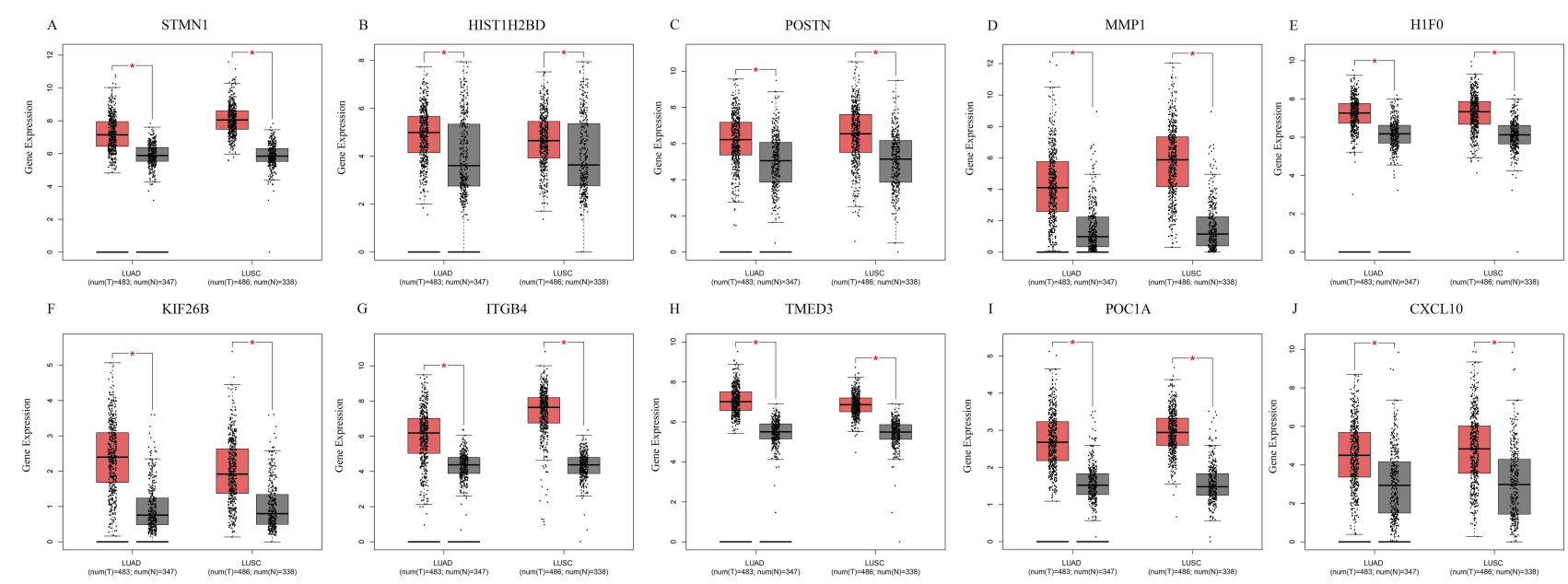

Figure 8 Expression of hub genes in non-small cell lung cancer and normal tissues, based on Gene Expression Profiling Interactive Analysis (GEPIA). (A) STMNI; (B) HISTIH2BD; (C) POSTN; (D) MMPI; (E) HIFO; (F) KIF26B; (G) ITGB4, (H) TMED3; (I) POCIA; (J) CXCLIO. 


\section{A POC1A}

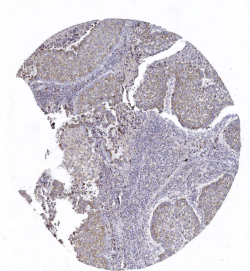

F STMN1

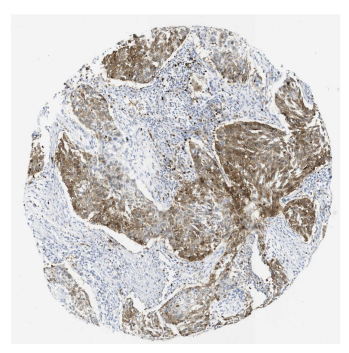

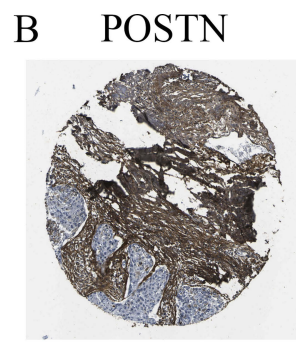

C H1F0

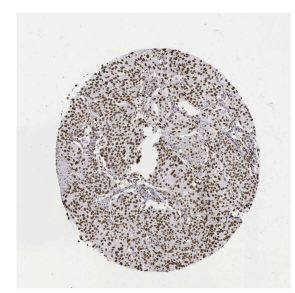

D HIST1H2BD

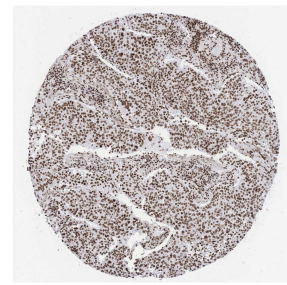

E KIF26B

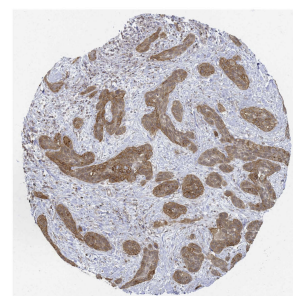

G TMED3

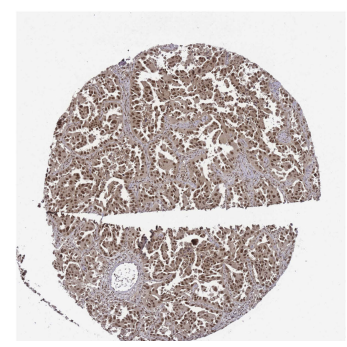

Figure 9 Validation of the ten hub genes on a translational level using the Human Protein Atlas database. (A) POCIA; (B) POSTN; (C) HIFO; (D) HISTIH2BD; (E) KIF26B; (F) STMNI; (G) TMED3.

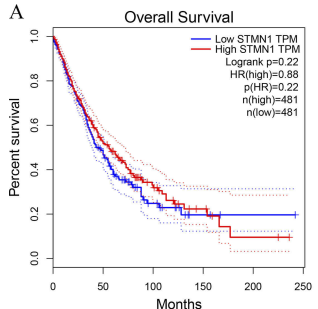

$\mathrm{F}$

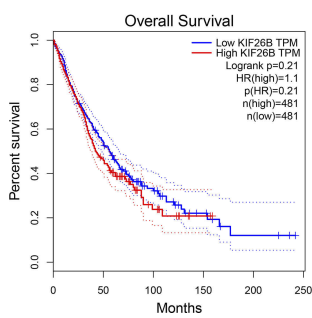

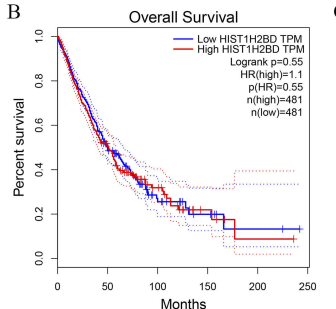

G

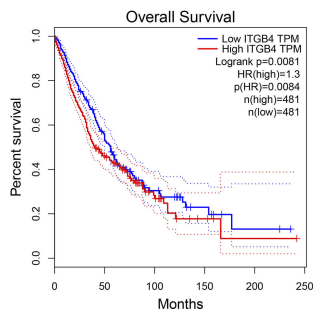

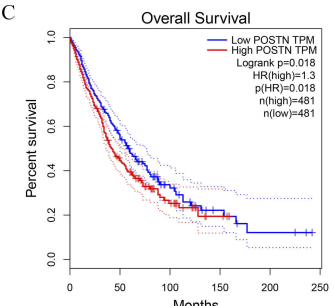

$\mathrm{H}$

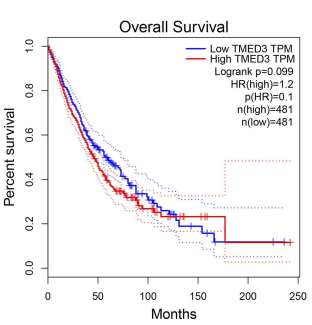

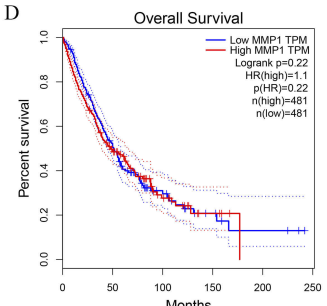

I

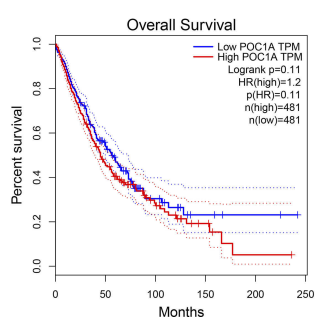

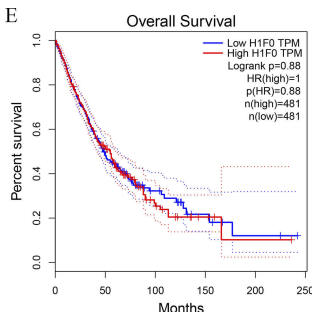

J

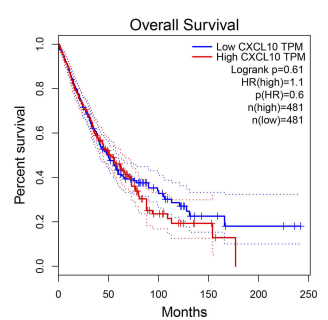

Figure 10 The association of hub gene expression and the survival in patients with non-small cell lung cancer. (A) STMNI; (B) HISTIH2BD; (C) POSTN; (D) MMPI; (E) HIFO;(F) KIF26B; (G) ITGB4; (H) TMED3; (I) POCIA; (J) CXCLIO.

(23\%, 10\%, and $10 \%$, respectively), and ITGB4 and KIF26B in LUSC (12\% and $11 \%$, respectively), including amplification and missense.

We then performed GO and KEGG pathway enrichment analyses of the most frequently altered neighboring genes. For LUAD, the enriched GO terms indicated that the genes were related to the Set1C/COMPASS complex, MLL3/4 complex, and histone methyltransferase complex. These proteins are primarily involved in beta-catenin-TCF complex assembly, positive regulation of chromosome organization, and positive regulation of gene silencing by miRNA (Figure 12A-C). Similarly, KEGG pathway analysis showed enrichment of the PI3K-Akt signaling pathway, focal adhesion, regulation of actin cytoskeleton, and cell cycle (Figure 12D). For LUSC, the enriched GO terms indicated that the genes were related to nuclear chromosome part, histone methyltransferase complex, and MLL3/4 complex. These proteins are primarily involved in beta-catenin-TCF complex assembly, vascular endothelial growth factor receptor signaling pathway, and axon guidance (Figure 12E-G). Similarly, KEGG pathway analysis showed enrichment in 
A

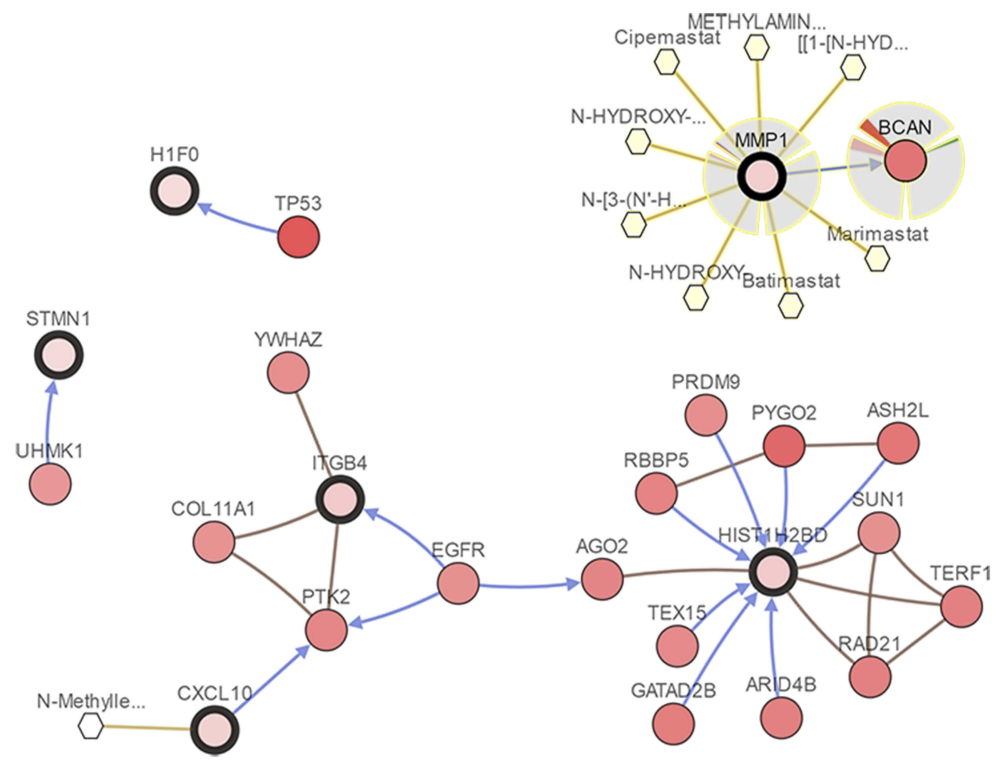

B

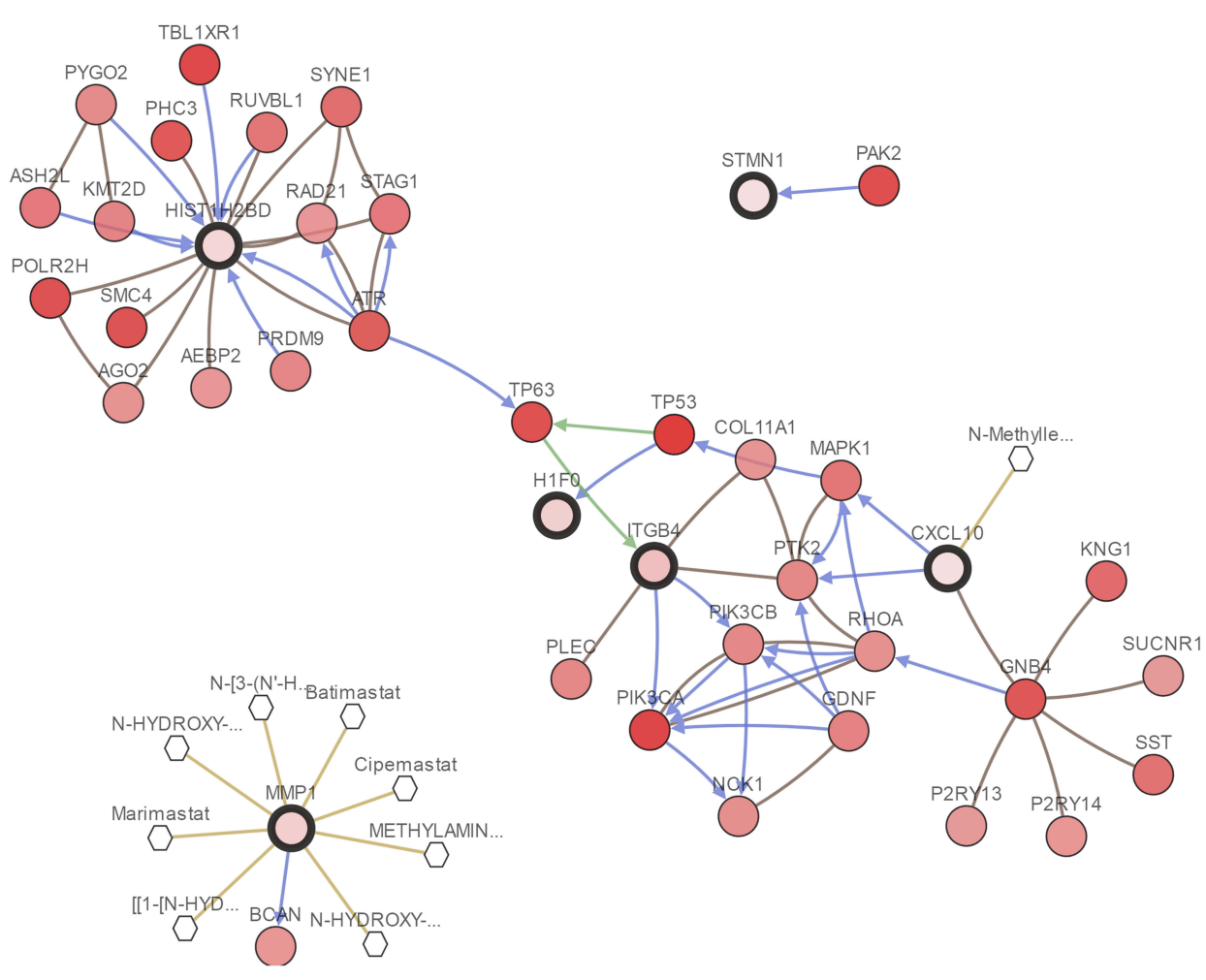

Figure I I Network review of hub genes neighborhood and genetic alteration of hub genes in non-small cell lung cancer. (A) Network review of hub genes neighborhood in LUAD. (B) Network review of hub genes neighborhood in LUSC. The relationship between hub genes and drugs is also exhibited.

chemokine signaling pathway, focal adhesion, and regulation of actin cytoskeleton (Figure 12H). The tab
OncoPrint displays an overview of genetic alterations in hub genes per sample. 


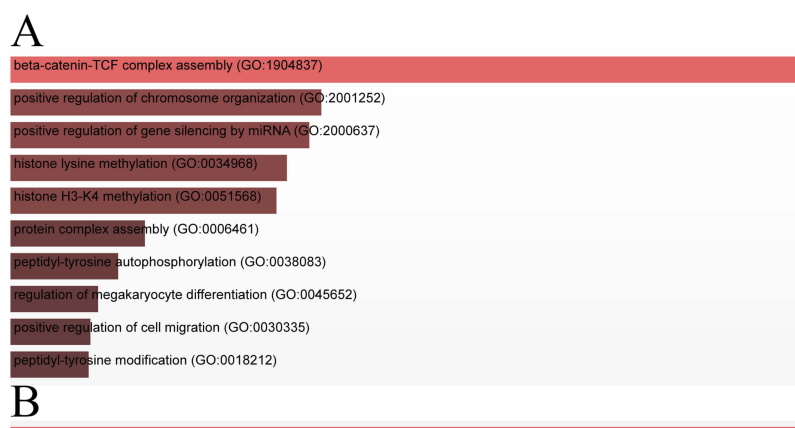

\section{$\mathrm{E}$}

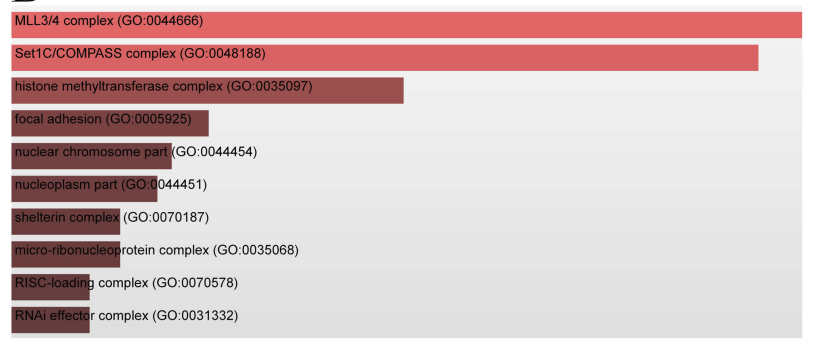

$\mathrm{C}$

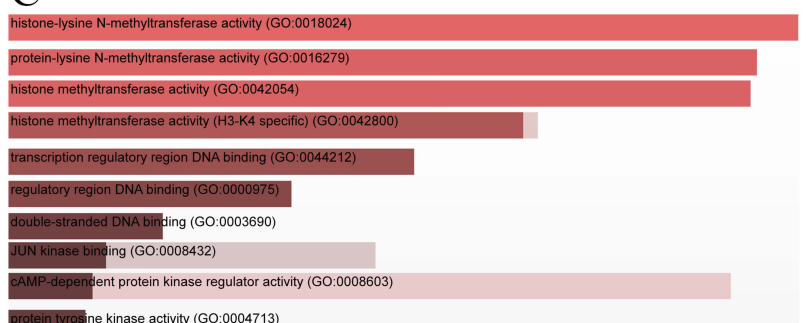

$\mathrm{D}$
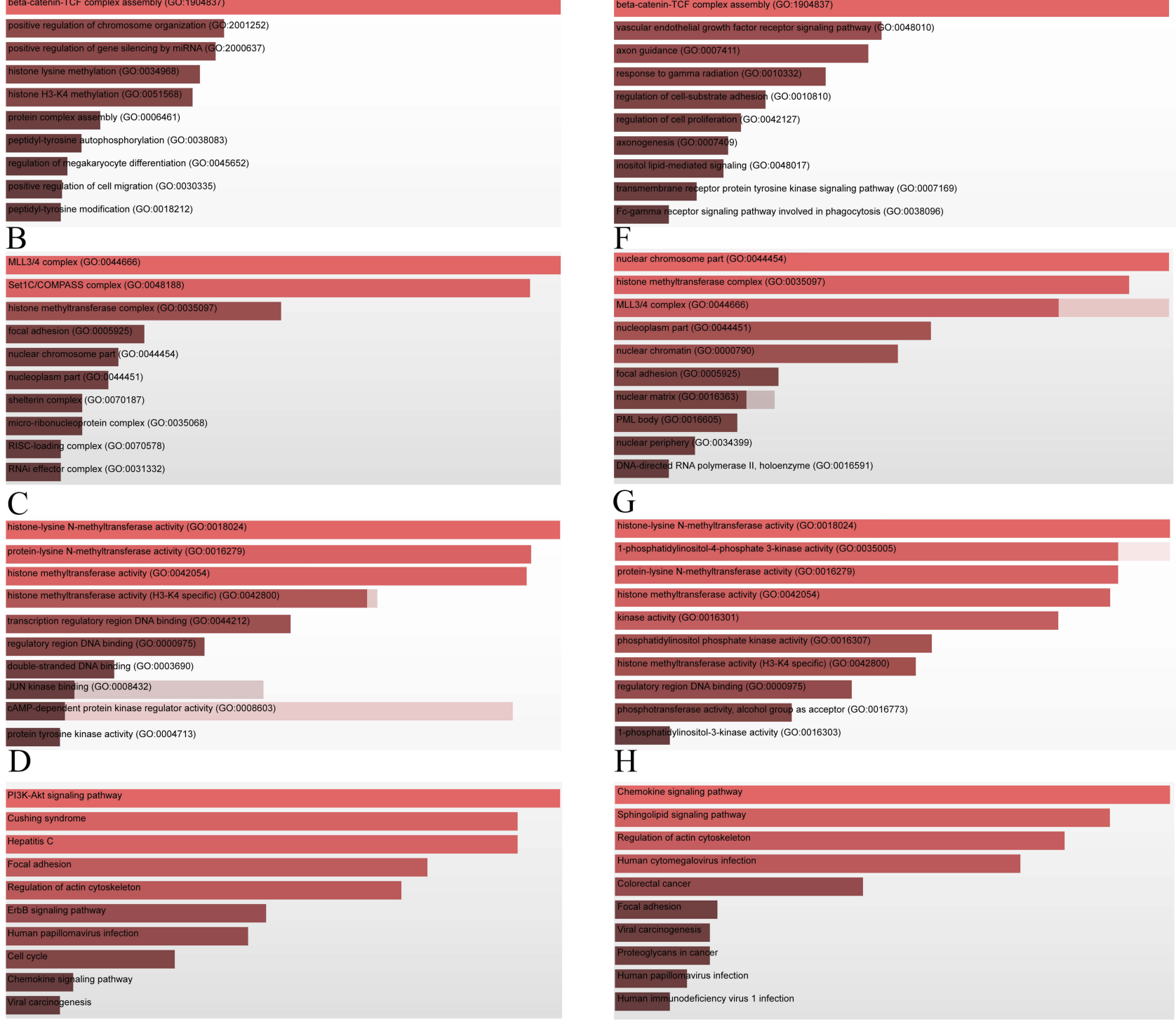

Fuder

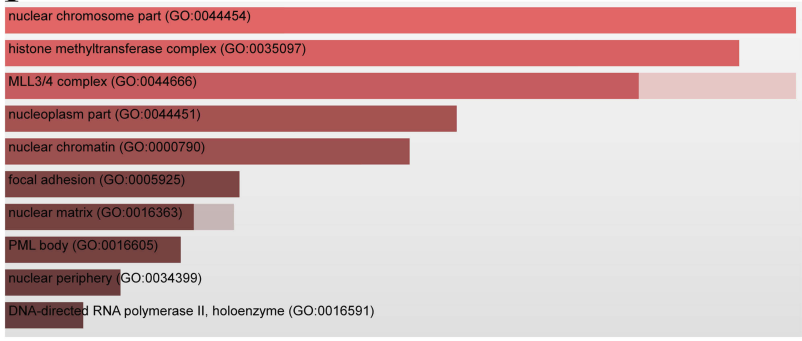

G

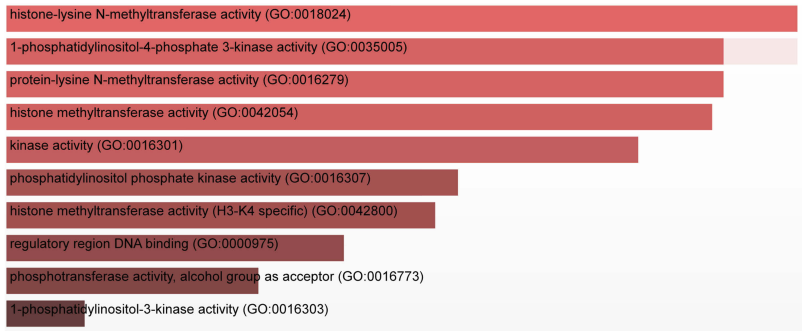

$\mathrm{H}$

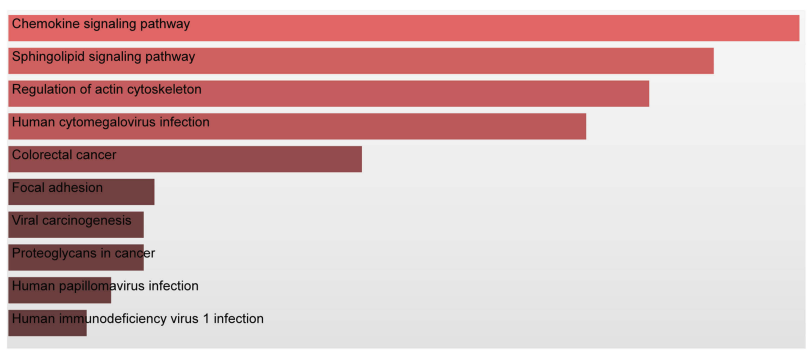

Figure 12 Enrichment analysis of the genes altered in the ten hub genes neighborhood in non-small cell lung cancer. (A) the top I0 enriched BP items in LUAD; (B) the top 10 enriched CC items LUAD; (C) the top 10 enriched MF items LUAD; (D) The top 10 enriched KEGG pathways LUAD; (E) the top 10 enriched BP items in LUSC; (F) the top 10 enriched CC items LUSC; (G) the top 10 enriched MF items LUSC; (H) The top 10 enriched KEGG pathways LUSC.

Identification Of Potential miRNA-mRNA Regulatory Pathways

A previous study showed that miR-133b inhibits cell growth of NSCLC by targeting EGFR and regulating its downstream signaling pathway. We found a positive correlation between EGFR, IGTB4, and PTK2 in NSCLC through the c-BioPortal database (Figure 13), suggesting that the miR-133b/EGFR/ ITGB4/FAK pathway may be present in NSCLC (Figure 14).

\section{Discussion}

Although the role of miRNAs in cancer has been postulated, the molecular mechanisms by which miRNAs regulate tumor metastasis have not been established. The aim of this study was to identify the expression of miR-133b in NSCLC and to study the association of clinical features of NSCLC with miR-133b. In addition, we investigated the possible mechanism of miR-133b in the metastasis of NSCLC.

In this study, we found that miR-133b was downregulated in NSCLC compared with normal tissues. For the TCGA data, miR-133b expression levels in LUAD and NSCLC were related to lymph node metastasis. For LUAD, we observed a significant difference in survival time between the downregulated and upregulated miR$133 \mathrm{~b}$ groups using the TCGA data. RT-qPCR analysis 
A
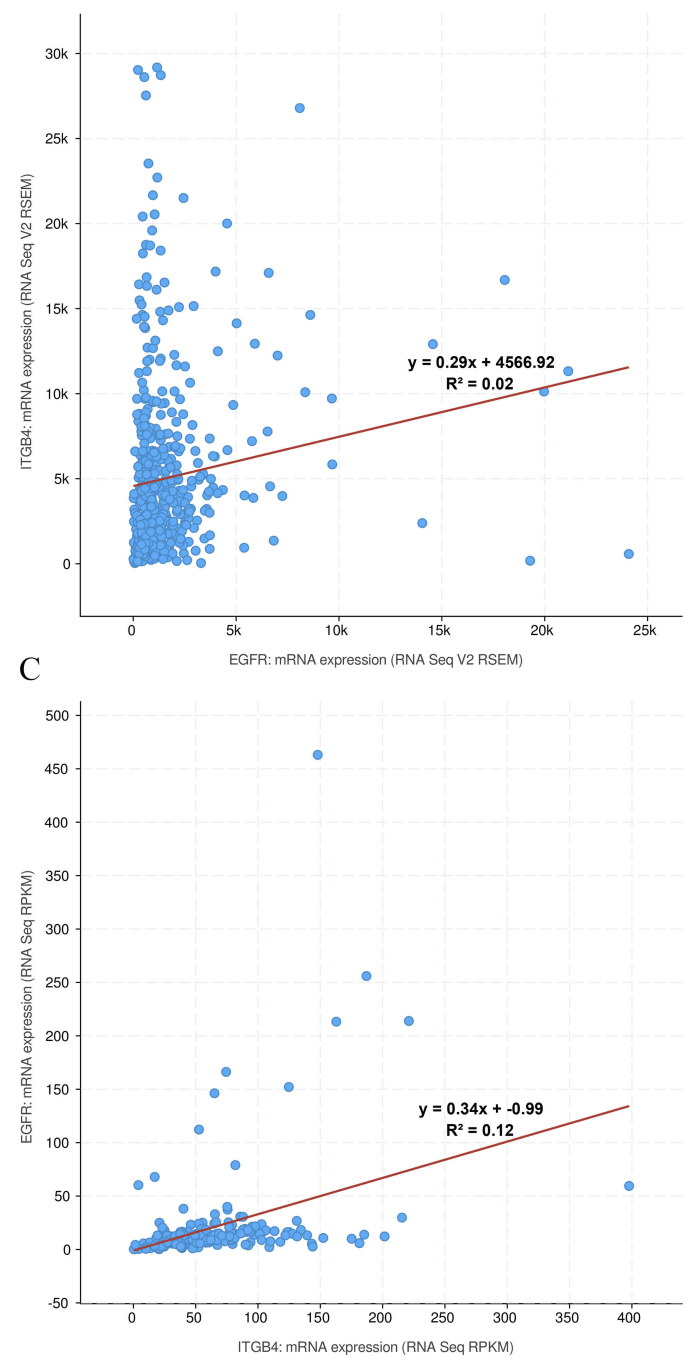

$\mathrm{B}$
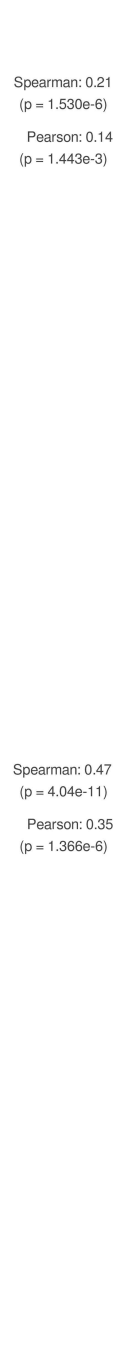
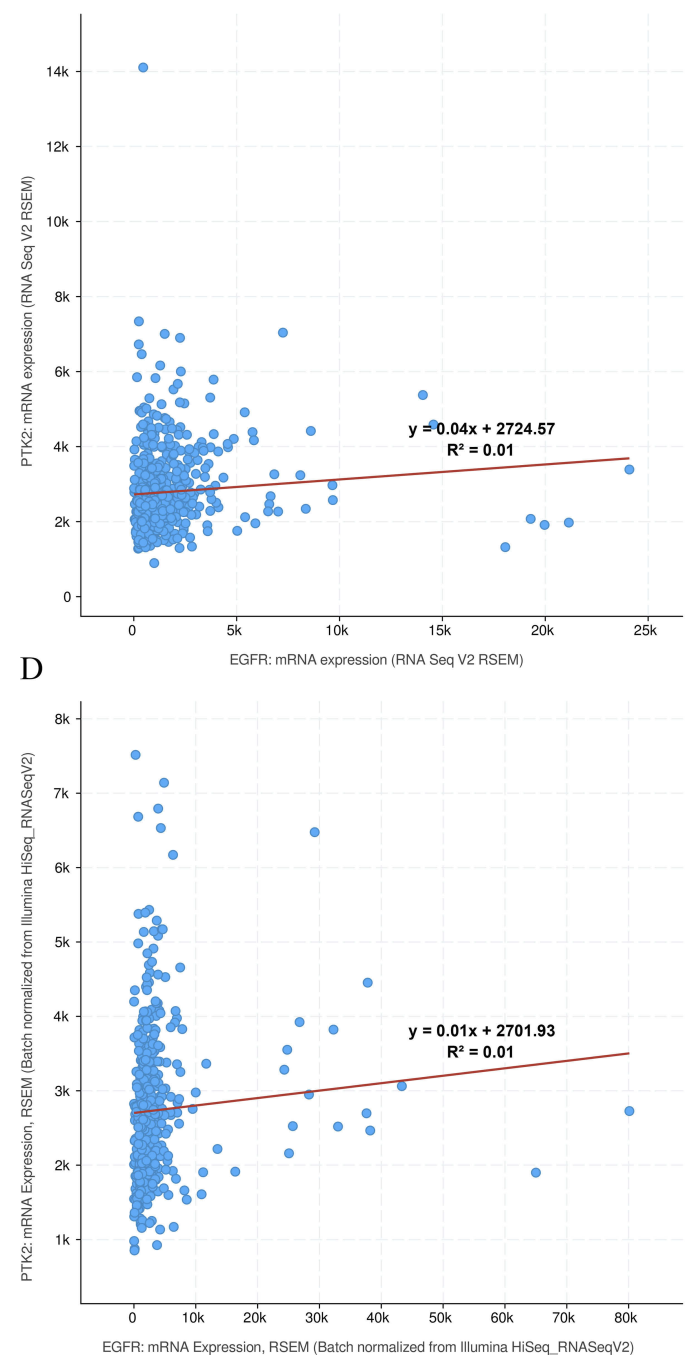

$(p=1.55 \mathrm{e}-7)$ Pearson: 0.09
$(p=0.0336)$

Figure 13 Correlation between expression of mRNA of EGFR, ITGB4 and PTK2 in TCGA HNSC patients. (A) Correlation between expression of mRNA of EGFR and ITGB4 in LUAD. (B) Correlation between expression of mRNA of EGFR and PTK2 in LUAD. (C) Correlation between expression of mRNA of EGFR and ITGB4 in LUSC. (D) Correlation between expression of mRNA of EGFR and PTK2 in LUSC. Pearson's coefficient less than 0.05 suggests significant significance.

also revealed that miR-133b expression was related to lymph node metastasis. These results indicate that miR$133 \mathrm{~b}$ could be involved in the metastasis of NSCLC. In addition, miR-133b might function as a biomarker for the diagnosis and prognosis in NSCLC.

Currently, the specific molecular mechanism of NSCLC is not widely understood. Therefore, bioinformatics analyses were performed to elucidate the possible mechanism of miR$133 \mathrm{~b}$ in NSCLC metastasis. We predicted the target genes using miRWalk3.0 and TCGA data. After intersection of the upregulated genes in LUAD and LUSC with the predicted target genes, 362 predicted target genes were selected. To further explore the roles of target genes, KEGG analysis was performed. We observed that some pathways were enriched with differentially expressed genes in NSCLC such as P53 signaling pathway and ECM-receptor interaction. In addition, KEGG pathway analysis of the most frequently altered neighbor genes showed enrichment of the PI3K-Akt signaling pathway, focal adhesion, regulation of actin cytoskeleton, and the cell cycle. These results of KEGG analysis revealed the roles of the candidate targets of miR-133b in NSCLC. Next, a PPI network was constructed and the top 10 central genes were identified. In addition, the GEPIA and Human Protein Atlas database were used to further assess expression analysis of these central genes in NSCLC.

Most of these hub genes have been identified as key regulators in NSCLC. For example, the periostin protein, encoded by the POSTN gene, is a component of the 


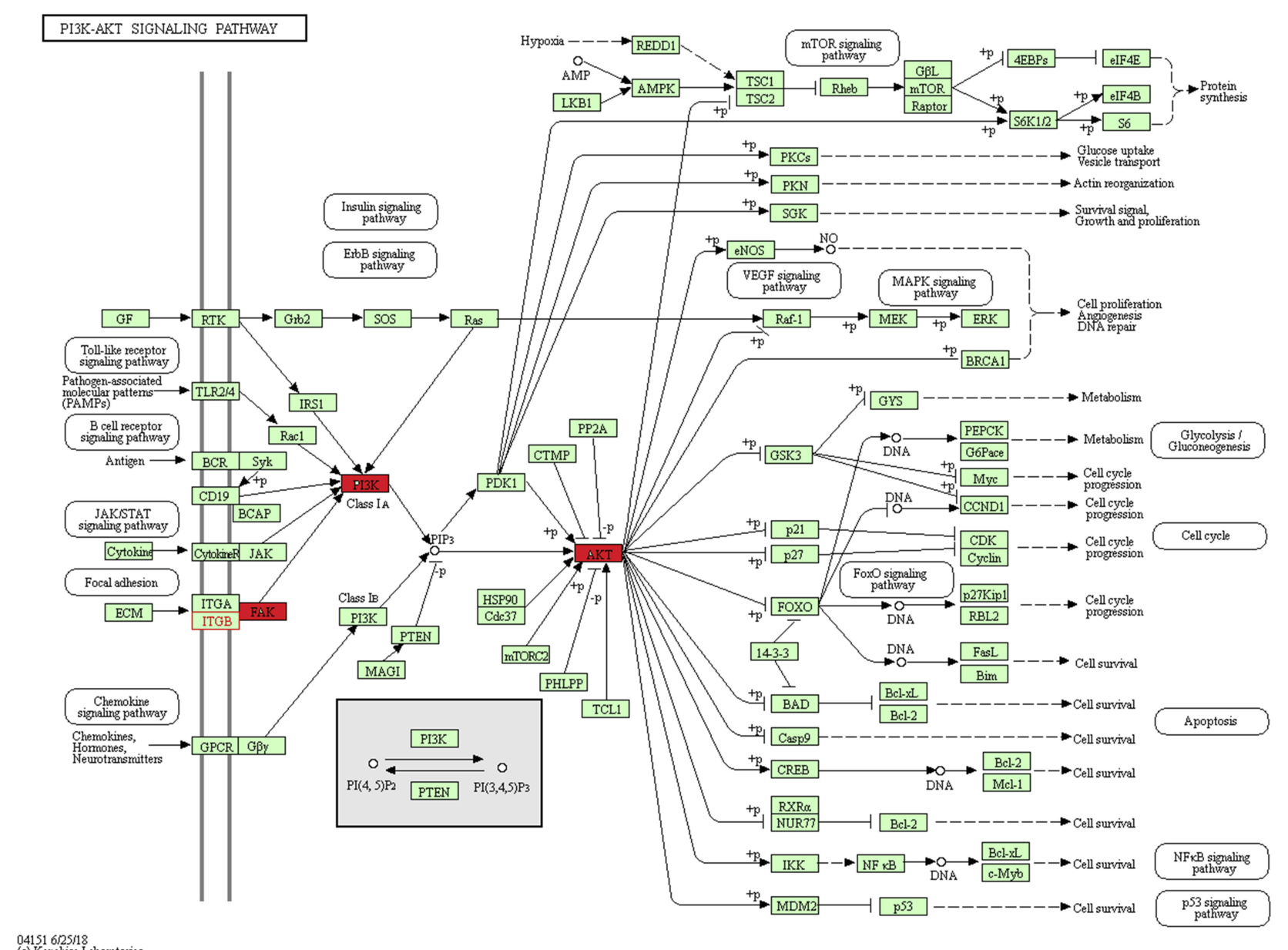

Figure 14 KEGG pathway annotations of the PI3K-Akt pathway in non-small cell lung cancer. Red marked nodes are associated with miR-133b.

extracellular matrix, which has been observed in a variety of human malignancies. ${ }^{11,12}$ Recently, periostin was identified as a novel factor in the growth, invasion, angiogenesis and metastasis of numerous types of tumors. Hu et al reported that periostin promotes epithelial-mesenchymal transition through the MAPK/miR-381 axis in lung cancer, thereby promoting lung cancer metastasis. ${ }^{13}$ Hong et al reported that overexpression of periostin predicts poor prognosis in NSCLC. ${ }^{14}$ In our study, overexpression of POSTN indicates poor prognosis using the TCGA data. In addition, Integrin $\alpha 6 \beta 4$ (ITGB4) is an extracellular matrix receptor that is involved in tumor progression. ${ }^{15,16}$ Stewart et al reported that integrin $\beta 4$ expression is elevated in LUSC and that its overexpression is associated with venous invasion and reduces overall survival in NSCLC patients. ${ }^{17}$ Based on the TCGA data, we confirmed that the expression of integrin $\beta 4$ is associated with poor prognosis. Zhu et al reported that the ITGB4/FAK/growth factor receptor-bound protein 2 (Grb2), protein kinase B (AKT), and extracellular signal-regulated kinase (ERK) pathways are involved in the regulatory mechanisms of miR-133b/EGFR axis in metastases in the esophageal squamous cell carcinoma. ${ }^{18}$ Liu et al reported that miR-133b specifically interacts with the 3'-UTR of EGFR mRNA in NSCLC. ${ }^{10}$ We found a positive correlation between EGFR, IGTB4, and PTK2 in NSCLC through the c-BioPortal database, suggesting that the miR-133b/EGFR/ ITGB4/FAK/PI3K/Akt pathway may also be involved in the pathogenesis of NSCLC. Based on the above studies, we have discovered the possible mechanism of miR-133b in NSCLC metastasis.

Taken together, the study validated that miR-133b is downregulated in NSCLC. In addition, miR-133b may potentially be used as a biomarker for diagnosis and prognosis in NSCLC. Bioinformatics analysis revealed that miR-133b could be involved in the metastasis of NSCLC. Additional experiments validating the above pathway are warranted. 


\section{Conclusions}

The study has shown that miR-133b is downregulated in NSCLC. In addition, miR-133b may be potentially utilized as a biomarker for the diagnosis and prognosis in NSCLC. Bioinformatics analysis revealed that miR-133b may be involved in the metastasis of NSCLC.

\section{Abbreviations}

$\mathrm{BP}$, Biological process; CC, Cellular component; GO, Gene Ontology; KEGG, Kyoto Encyclopedia of Genes and Genomes; MF, Molecular function; NSCLC, Nonsmall cell lung cancer; LUAD, Lung Adenocarcinoma; LUSC, Lung squamous cell carcinoma; PPI, Protein-protein interaction network; qRT-PCR, Quantitative realtime PCR; TCGA, the Cancer Genome Atlas.

\section{Ethics Approval And Consent To Participate}

The study methodologies conformed to the standards set by the Declaration of Helsinki, and the study was approved by the Ethics Committee of the Affiliated Tumor Hospital of Guangxi Medical University. Written informed consent was obtained from the patients to utilize their tissue samples.

\section{Availability Of Data And Material}

The datasets supporting the conclusions of this article are included within the article and its additional files. A total of 44 paired non-small cell lung cancer specimens were obtained from the Affiliated Tumor Hospital of Guangxi Medical University.

\section{Acknowledgment}

We thank LetPub for its linguistic assistance during the preparation of this manuscript.

\section{Author Contributions}

Lin Ruan and Guan-yu Chen were major contributors in the conception and design, acquisition of data, analysis and interpretation of data, drafting the article, and gave final approval of the version to be published. Lin Ruan and Guan-yu Chen agree to be responsible for all aspects of the work in ensuring that questions related to the accuracy or integrity of any part of the work are appropriately investigated and resolved.

\section{Disclosure}

The authors report no conflicts of interest in this work.

\section{References}

1. Bray F, Ferlay J, Soerjomataram I, Siegel RL, Torre LA, Jemal A. Global cancer statistics 2018: GLOBOCAN estimates of incidence and mortality worldwide for 36 cancers in 185 countries. $C A$ : A Cancer Journal for Clinicians. 2018;68(6):394-424. doi:10.3322/ caac. 21492

2. Hirsch FR, Scagliotti GV, Mulshine JL, et al Lung cancer: current therapies and new targeted treatments. Lancet. 2017;389(10066):299-311.

3. Yun YH, Kim YA, Sim JA, et al Prognostic value of quality of life score in disease-free survivors of surgically-treated lung cancer. $B M C$ Cancer. 2016;16(1):505.

4. Beermann J, Piccoli MT, Viereck J, Thum T. Non-coding RNAs in development and disease: background, mechanisms, and therapeutic approaches. Physiol Rev. 2016;96(4):1297-1325.

5. Lin $\mathrm{K}, \mathrm{Xu} \mathrm{T}, \mathrm{He} \mathrm{BS}$, et al MicroRNA expression profiles predict progression and clinical outcome in lung adenocarcinoma. Onco Targets Ther. 2016;9:5679-5692.

6. Zhou Q, Huang SX, Zhang F, et al MicroRNAs: a novel potential biomarker for diagnosis and therapy in patients with non-small cell lung cancer. Cell Proliferation. 2017;50:e12394. doi:10.1111/cpr.12394

7. Cheng Y, Jia B, Wang Y, Wan S. miR-133b acts as a tumor suppressor and negatively regulates ATP citrate lyase via PPARgamma in gastric cancer. Oncol Rep. 2017;38(5):3220-3226.

8. Wang $\mathrm{X}, \mathrm{Bu} \mathrm{J}$, Liu X, et al miR-133b suppresses metastasis by targeting HOXA9 in human colorectal cancer. Oncotarget. 2017;8 (38):63935-63948

9. L Yang, J Hou, X-H Cui, L-N Suo, Y-W Lv, et al MiR-133b regulates the expression of CTGF in epithelial-mesenchymal transition of ovarian cancer. Eur Rev Med Pharmacol Sci. 2017;21(24):56025609

10. Liu L, Shao X, Gao W, et al MicroRNA-133b inhibits the growth of non-small-cell lung cancer by targeting the epidermal growth factor receptor. FEBS J. 2012;279(20):3800-3812.

11. Utispan K, Thuwajit P, Abiko Y, et al Gene expression profiling of cholangiocarcinoma-derived fibroblast reveals alterations related to tumor progression and indicates periostin as a poor prognostic marker. Mol Cancer. 2010;9(1):13.

12. Zhang Y, Zhang G, Li J, Tao Q, Tang W. The expression analysis of periostin in human breast cancer. $J$ Surg Res. 2010;160(1):102-106.

13. Hu WW, Chen PC, Chen JM, et al Periostin promotes epithelialmesenchymal transition via the MAPK/miR-381 axis in lung cancer. Oncotarget. 2017;8(37):62248-62260.

14. Hong L-Z, Wei X-W, Chen J-F, Shi Y. Overexpression of periostin predicts poor prognosis in non-small cell lung cancer. Oncol Lett. 2013;6(6):1595-1603. doi:10.3892/ol.2013.1590

15. Guo W, Giancotti FG. Integrin signalling during tumour progression. Nature Rev Mol Cell Bio. 2004;5(10): 816

16. Stewart RL, O'Connor KL. Clinical significance of the integrin alpha6beta4 in human malignancies. Lab Invest 2015;95(9): 976-986.

17. Stewart RL, West D, Wang C, et al Elevated integrin $\alpha 6 \beta 4$ expression is associated with venous invasion and decreased overall survival in non-small cell lung cancer. Human Pathol. 2016;54:174-183. doi:10.1016/j.humpath.2016.04.003

18. Zhu JF, Liu Y, Huang H, et al MicroRNA-133b/EGFR axis regulates esophageal squamous cell carcinoma metastases by suppressing anoikis resistance and anchorage-independent growth. Cancer cell Int 2018;18(1):193. 


\section{Publish your work in this journal}

OncoTargets and Therapy is an international, peer-reviewed, open access journal focusing on the pathological basis of all cancers, potential targets for therapy and treatment protocols employed to improve the management of cancer patients. The journal also focuses on the impact of management programs and new therapeutic

Submit your manuscript here: https://www.dovepress.com/oncotargets-and-therapy-journal agents and protocols on patient perspectives such as quality of life, adherence and satisfaction. The manuscript management system is completely online and includes a very quick and fair peer-review system, which is all easy to use. Visit http://www.dovepress.com/ testimonials.php to read real quotes from published authors. 